\title{
Infrastructure requirement of knowledge management system model of statistical learning tool (SLT) for education community
}

\begin{abstract}
Today, teaching and learning (T\&L) using technology as tool is becoming more important especially in the field of statistics as a part of the subject matter in higher education system environment. Eventhough, there are many types of technology of statistical learnig tool (SLT) which can be used to support and enhance T\&L environment, however, there is lack of a common standard knowledge management as a knowledge portal for guidance especially in relation to infrastructure requirement of SLT in servicing the community of user (CoU) such as educators, students and other parties who are interested in performing this technology as a tool for their T\&L. Therefore, there is a need of a common standard infrastructure requirement of knowledge portal in helping $\mathrm{CoU}$ for managing of statistical knowledge in acquiring, storing, desseminating and applying of the statistical knowedge for their specific purposes. Futhermore, by having this infrastructure requirement of knowledge portal model of SLT as a guidance in promoting knowledge of best practise among the $\mathrm{CoU}$, it can also enhance the quality and productivity of their work towards excellence of statistical knowledge application in education system environment.
\end{abstract}

Keyword: Knowledge management system; Knowledge portal; Teaching and learning; Statistical learning tool; Community of user 